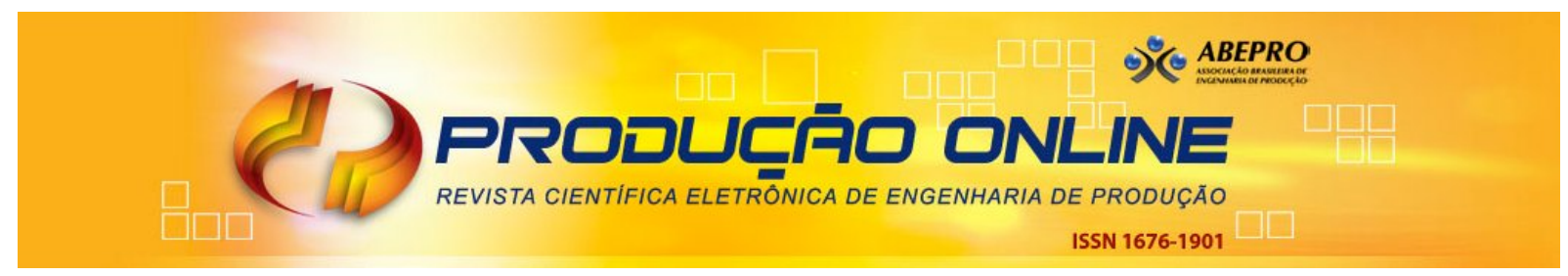

\title{
EFICÁCIA DA HEURÍSTICA DA UTILIZAÇÃO INCREMENTAL PARA BALANCEAMENTO DE LINHA: ESTUDO DE CASO EM UM PROCESSO DE TRATAMENTO DE SUPERFÍCIE
}

\section{EFFECTIVENESS OF INCREMENTAL UTILIZATION HEURISTIC FOR ASSEMBLY LINE BALANCING: A CASE STUDY IN SURFACE TREATMENT PROCESS}

\author{
Marcela de Souza Leite* E-mail: marcelaleiteengenheira@gmail.com \\ Stephanie Conceição Santos* E-mail: stephaniesan 09@hotmail.com \\ Adriano Maniçoba da Silva* E-mail: adrianoms@ifsp.edu.br \\ William de Paula Ferreira**E-mail: william.ferreira@ifsp.edu.br \\ *Instituto Federal de Educação, Ciência e Tecnologia de São Paulo (IFSP), São Paulo, SP \\ **Escola Politécnica da Universidade de São Paulo (Poli-USP), São Paulo, SP
}

\begin{abstract}
Resumo: O balanceamento de linha permite aumentar a eficiência de um processo produtivo. Apesar de recorrentemente pesquisada no exterior, a aplicação da heurística da utilização incremental para balanceamento de linha no Brasil foi apresentada em poucos estudos. Neste contexto, o objetivo desta pesquisa é, através de um estudo de caso, apresentar o resultado do balanceamento das atividades da linha de tratamento de superfície de uma indústria de mobiliário escolar e desportivo. Mediante a aplicação da heurística da utilização incremental para o balanceamento de linha, foi possível ampliar a capacidade produtiva do setor de serralheria. Comparou-se ainda a solução obtida pela heurística com a solução ótima obtida com programação linear. Concluiu-se que a aplicação da heurística de utilização incremental é eficaz para realizar o balanceamento de linha podendo ser mais bem explorada em aplicações no âmbito nacional.
\end{abstract}

Palavras-chave: Balanceamento de Linha. Heurística. Utilização incremental. Programação Linear.

Tratamento de superfície.

Abstract: The line balancing can increase the efficiency of a production process. Although repeatedly searched abroad, heuristic applying the incremental use for line balancing in Brazil was presented in a few studies. In this context, the aim of this study is to present, thought a case study, the result of balancing the activities of the surface treatment line of a school and sports furniture industry. By applying the heuristic of incremental utilization for line balancing was possible to expand the production capacity of the sawmill industry. It also compared the solution obtained by the heuristic with the optimal solution obtained with linear programming. It was concluded that the application of the incremental use of heuristics is effective to carry out line balancing can be best exploited in applications nationwide.

Keywords: Line balancing. Heuristic. Incremental utilization. Surface treatment. Linear programming.

Revista Produção Online. Florianópolis, SC, v.17, n. 3, p. 781-803, 2017. 


\section{INTRODUÇÃO}

O layout por produto, também conhecido como linha de produção, consiste em estações de trabalho ou departamentos dispostos em uma sequência linear (MAKE et al, 2017). Segundo Kumar (2013), o problema fundamental de uma linha de montagem é atribuir tarefas a um número de estações de trabalho de maneira tão igualitária quanto possível, pois, desta forma, é possível aumentar a eficiência do processo produtivo. O balanceamento de linha de produção busca otimizar e sincronizar os recursos necessários para o processamento de um produto ou serviço, de forma a atender a demanda no tempo previsto (FERNANDES; DALALIO, 2000).

Apesar do desenvolvimento na década de 1950, o balanceamento de linha de montagem ainda é um tema atual, reforçando a importância de se aumentar a produtividade pela divisão do trabalho. Silva et al. (2014) revisaram as principais publicações realizadas nos últimos 50 anos referentes a linhas de produção, sendo evidenciado que o balanceamento de linhas produtivas figurava entre os temas mais pesquisados. Segundo estes mesmos autores, quando analisada a literatura nacional sobre o tema, verifica-se, entretanto, que poucos estudos têm explorado este tema, sugerindo que o mesmo seja objeto de pesquisas futuras, com a exploração de problemas e casos reais, através da utilização de métodos como estudos de caso, estudos de campo, pesquisa-ação e levantamentos. Uma pesquisa realizada em bases de dados nacionais verificou que a heurística da utilização incremental para o balanceamento de linhas produtivas foi investigada apenas no estudo de Breginski (2013).

O balanceamento de linha pode ser feito por modelos otimizantes (modelos matemáticos), heurísticos, metaheurísticos ou simulação (SCHOLL; BECKER, 2006). Métodos heurísticos, ou que utilizam regras simples, tal como o método investigado neste estudo, têm sido usados para desenvolver soluções satisfatórias para o problema de balanceamento de linha.

O objetivo deste estudo é apresentar os resultados do balanceamento das atividades da linha de tratamento de superfície de uma indústria de mobiliário escolar e 
desportivo, com a técnica da heurística da utilização incremental e comparar esta solução com a desenvolvida por otimização linear. A partir das lacunas indicadas nos parágrafos precedentes, este estudo parte da definição da seguinte pergunta problema: Qual é a eficácia da heurística da utilização incremental para balanceamento de uma linha de produção com layout por produto?

\section{REVISÃO DA LITERATURA}

\subsection{Arranjo produtivo e balanceamento de linha}

Segundo Silva e Rentes (2012), o layout ou arranjo físico do setor de produção de uma organização pode ser definido como a localização e a distribuição espacial dos recursos produtivos tais como máquinas, equipamentos, pessoas, e instalações, no ambiente operacional e verifica ainda como esta distribuição se relaciona com o desempenho da unidade produtiva.

Apesar de estudos anteriores apresentarem uma série de tipos de arranjos físicos, quatro tipos têm sido mais evidenciados por incorporarem em seus modelos amplo escopo de aplicação. Segundo Sivasankaran e Shahabudeen (2014), os arranjos físicos mais pesquisados, e com conceitos consolidados no ambiente industrial são: 1) arranjo físico posicional; 2) arranjo físico por produto; 3) arranjo físico funcional e; 4) arranjo físico celular. Esses arranjos físicos relacionam-se com os tipos de processo descritos na matriz produto processo, conforme apresentado em Santos (2014).

O arranjo físico por produto é justificado quando o processo deve atender a um maior nível de demanda, sendo este nível relativamente estável, em produto padronizado. No arranjo celular há um nível intermediário tanto de demanda quanto de variedade de materiais no processo produtivo. Já o arranjo físico funcional é usado quando a variedade de peças é maior e o volume de produção não é suficiente para justificar um arranjo físico por produto ou celular. Por fim, no arranjo posicional, a

demanda pode apresentar seu menor nível e há necessidade de customizar o produto (DRIRA; HAJRI-GABOUJ; PIERREVAL, 2007).

Revista Produção Online. Florianópolis, SC, v.17, n. 3, p. 781-803, 2017. 
Para Tompkins et al. (2003), dentre as vantagens de se utilizar um layout por produto estão: planejamento e controle simplificado da produção; menor nível de estoques intermediários; tempo de produção unitária reduzido e; tarefas que costumam ser realizadas são relativamente simples e requerem menor nível de treinamento para sua execução.

De acordo Kumar (2013), no arranjo físico por produto, os recursos físicos transformadores estão localizados em sequência especificamente para atender às necessidades de fluxo contínuo dos produtos e seus componentes. O projeto detalhado do arranjo físico por produto inclui um número de decisões como o tempo de ciclo que, direcionará outros parâmetros do processo, o número de estágios da operação, a forma como as tarefas são alocadas aos estágios na linha e, o arranjo dos estágios na linha de produção. A alocação de tarefas nos estágios, de modo que se busque a taxa máxima de fluxo, é chamada balanceamento de linha.

Cada tarefa é definida como uma quantidade de trabalho indivisível e possui um tempo de operação associado. Deste modo, o balanceamento de linha consiste em distribuir e nivelar o tempo total das operações em relação ao tempo homem-máquina, de modo a maximizar as saídas do processo em cada estágio de uma linha de produção (BOYSEN et al., 2007).

A meta do balanceamento de linha é empregar eficientemente os recursos produtivos. O principal objetivo é minimizar o tempo ocioso, equivalente a encontrar o número mínimo de estações de trabalho dado um tempo de ciclo desejado (REGINATO, 2014). O balanceamento também se faz necessário quando existem alterações do processo, como a inclusão ou exclusão de tarefas, modificações em componentes, alterações no tempo de processamento e também na implantação de novos processos (FARNES; PEREIRA, 2006, SNATKIN et al, 2015).

Gosh e Gagnon (1989) relatam que, a primeira publicação a analisar o balanceamento de linha foi realizada por Helgeson et al. (1954), enquanto que se atribui a Salveson (1955) a primeira publicação contendo uma formulação matemática seguida de uma sugestão de solução por meio de programação linear.

Revista Produção Online. Florianópolis, SC, v.17, n. 3, p. 781-803, 2017. 
A literatura divide os problemas de balanceamento de linha em duas categorias: problema de balanceamento de linha de montagem simples (SALBP - Simple Assembly Line Balancing Problem) e problemas de linha de montagem generalizado (GALBP Generalized Assembly Line Balancing Problem).

O SALBP trata do balanceamento de uma linha serial monoproduto. Este modelo possui duas variantes: o SALBP-1, cujo objetivo é minimizar o número de estações de trabalho necessárias para alocação de todas as tarefas, respeitando as relações de precedência e o tempo de ciclo de operação da linha; e o SALBP-2, que minimiza o tempo de ciclo, sujeito a restrições de precedência e um número máximo de estações disponíveis (SILVA JUNIOR, MIRANDA JUNIOR; CONCEIÇÃO, 2010).

De acordo com Boysen et al. (2007) o SALBP se baseia num conjunto de hipóteses que limitam as aplicações do modelo, bem como sua utilidade para resolução de problemas específicos. As hipóteses são apresentadas no Quadro 1.

\begin{tabular}{|c|c|}
\hline HIPÓTESE & DESCRIÇÃO \\
\hline Homogeneidade & Produção em massa de produtos homogêneos \\
\hline Processamento uniforme & $\begin{array}{l}\text { Todas as tarefas são processadas em uma forma pré- } \\
\text { determinada (não mais que uma alternativa de processamento) }\end{array}$ \\
\hline Tempos de ciclo iguais & $\begin{array}{l}\text { Os tempos de ciclo de todos os postos da linha são iguais e } \\
\text { definidos de acordo com uma quantidade desejada de output }\end{array}$ \\
\hline Processo sequencial & $\begin{array}{l}\text { A linha segue uma sequência e não apresenta linhas } \\
\text { alimentadoras ou elementos paralelos }\end{array}$ \\
\hline Precedência das tarefas & $\begin{array}{l}\text { A sequência de processamento das tarefas está sujeita a } \\
\text { restrições de precedência }\end{array}$ \\
\hline $\begin{array}{l}\text { Precisão dos tempos das } \\
\text { tarefas }\end{array}$ & O tempo de tarefa é determinístico \\
\hline Atribuição livre das tarefas & $\begin{array}{l}\text { Não há restrições de atribuição de tarefas, além das restrições de } \\
\text { precedência }\end{array}$ \\
\hline Indivisibilidade das tarefas & Uma tarefa não pode ser repartida entre dois ou mais postos \\
\hline Postos multitarefa & $\begin{array}{l}\text { Todos os postos são igualmente equipados com relação às } \\
\text { máquinas e operários }\end{array}$ \\
\hline
\end{tabular}

Fonte: Adaptado de Boysen et al. (2007)

As hipóteses elencadas no Quadro 1 têm como objetivo facilitar a modelagem e solução de problemas de balanceamento de linha. Essas hipóteses simplificam o 
problema de configuração da linha de montagem ao problema central de designar tarefas a postos.

Quanto às técnicas de balanceamento de linha, Gaither e Frazier (2012) afirmam que os pesquisadores têm usado a programação linear, a programação dinâmica e outros modelos matemáticos para estudar problemas de balanceamento de linha, apesar dos métodos heurísticos terem sido utilizados para desenvolver soluções satisfatórias para o problema de balanceamento. Apesar de nem sempre serem ótimas, as soluções derivadas de métodos heurísticos reduzem a necessidade de sofisticação e utilização de recursos computacionais complexos.

De acordo com Cristo (2010), grande parte das heurísticas de balanceamento apoia-se em uma lista ordenada de atividades nas quais um alocador busca uma solução. Em geral, são três os principais passos dos algoritmos de solução: 1) classificação das tarefas para criação da lista; 2) aplicação do alocador e; 3) análise dos resultados. $\mathrm{O}$ autor destaca as seguintes heurísticas utilizadas para 0 balanceamento de linha de montagem: método dos pesos posicionais, método de Kilbridge e Wester e regra do maior candidato, as quais são geralmente aplicadas em linhas uni-modelo.

Breginski (2013) realizou o balanceamento de linha de montagem utilizando oito heurísticas diferentes: 1) regra heurística da tarefa de mais longa duração; 2) heurística do número de tarefas imediatamente subsequentes 3) COMSOAL; 4) heurística da utilização incremental; 5) peso posicional; 6) peso posicional Invertido; 7) número de tarefas sucessoras; e 8) Número de tarefas predecessoras. O autor encontrou desempenhos diferentes, mas com pouca variação entre os métodos comparados.

A próxima seção aborda a heurística da utilização incremental.

\subsection{Heurística da utilização incremental}

Gaither e Frazier (2012) afirmam que a vantagem da regra heurística incremental é a capacidade deste procedimento resolver problemas de balanceamento de linha independentemente da duração das tarefas em relação ao tempo de ciclo total. Os 
autores descrevem ainda que a regra simplesmente acrescenta tarefas a uma estação de trabalho em ordem de precedência de tarefa, uma de cada vez, até que a utilização seja de $100 \%$ ou se observe que tal utilização decresça. Então, esse procedimento é repetido na estação seguinte para as tarefas restantes.

Figura 1 - Algoritmo da heurística da utilização incremental

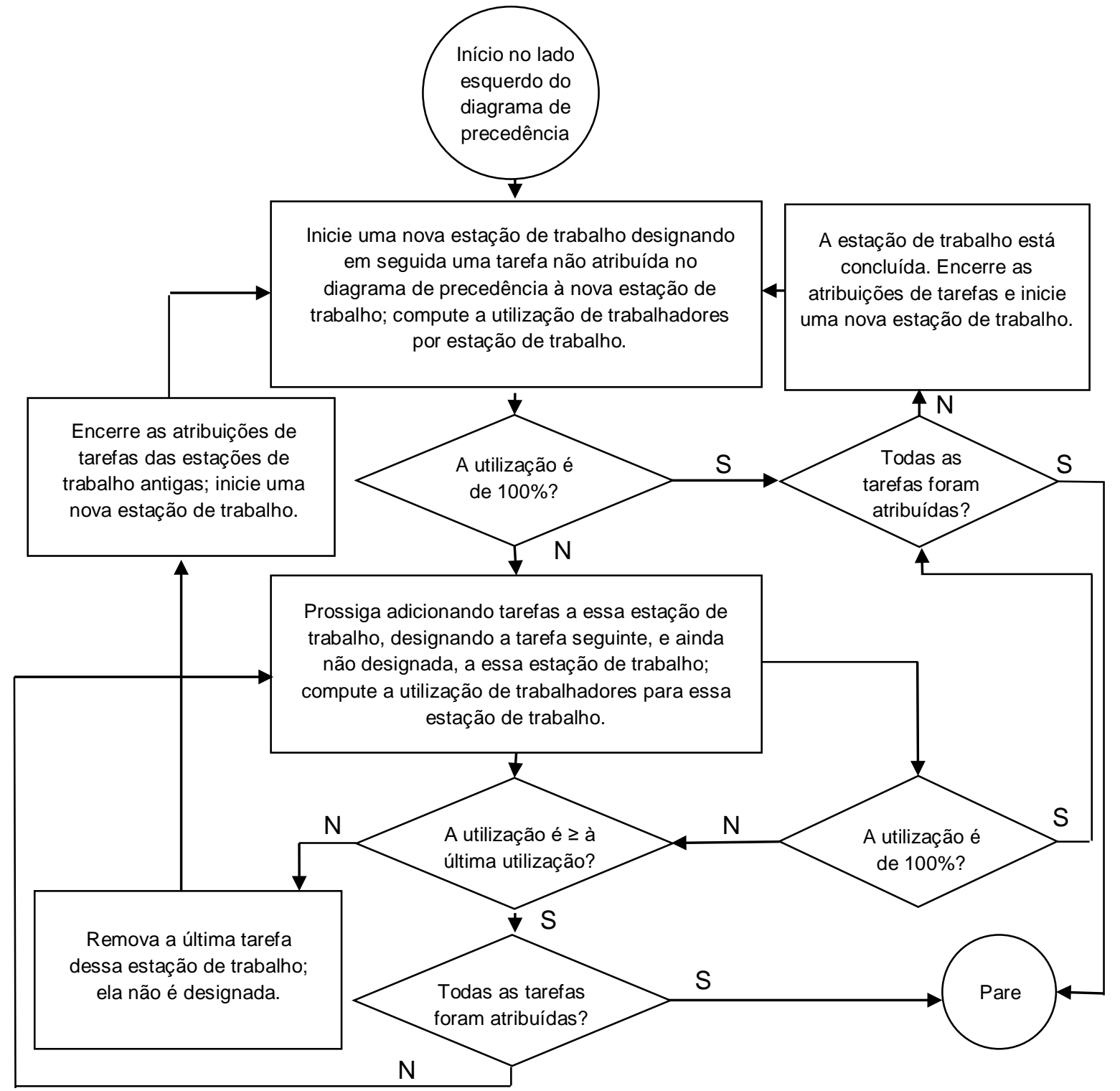

Fonte: Adaptado de Gaither e Frazier (2012, p. 211)

Revista Produção Online. Florianópolis, SC, v.17, n. 3, p. 781-803, 2017. 
A heurística da utilização incremental é apresentada na Figura 1. Quando o volume de produção é definido, o tempo de ciclo pode ser calculado e, esse valor condiciona os outros parâmetros utilizados pelas regras heurísticas de balanceamento de linha que determinam o número de estações de trabalho necessário (GAITHER; FRAZIER, 2012).

É necessário ainda conhecer o processo no qual se aplicará o balanceamento de linha. Conforme Boysen et al (2007), o diagrama de precedência resume convenientemente as informações chaves do processo tal como a contribuição de cada estação de trabalho ao produto final, a ordem com que as tarefas devem ser realizadas e as relações de precedência.

O exame da Figura 1 permite verificar as etapas envolvidas no balanceamento de linha. Para Davis, Aquilano e Chase (2001), existem seis etapas envolvidas no balanceamento de linhas de produção uni-produtos para que o mesmo seja implantado com eficácia:

1) Especificação da relação sequencial entre as tarefas, utilizando um diagrama de precedência. O diagrama, na sua versão simplificada, consiste de círculos e setas. Os círculos representam tarefas individuais e as setas indicam sua ordem na sequência do processo produtivo (TELO et al, 2017).

2) Determinação do tempo de ciclo necessário. De acordo com Oliveira (2017), o tempo de ciclo é um dos principais elementos para realizar o balanceamento de linha. Ele é calculado considerando a demanda do tempo provável dos produtos e a quantidade de tempo disponível para a produção durante o mesmo intervalo. O tempo de ciclo pode ser calculado de acordo com a Equação 1:

$$
c=\frac{\text { Tempo disponível de produção }}{\text { Demanda }}
$$

3) Determinação do número mínimo teórico de estações de trabalho. Para Martins e Laugeni (2015) o número mínimo teórico de estações é a quantidade 
mínima teórica de operadores necessários para atingir determinada produção. O cálculo deste número é apresentado na Equação 2:

$$
M T=\frac{\text { Tempo total para produzir uma peça na linha }}{\text { Tempo de ciclo }}
$$

4) Seleção de uma regra básica na qual as tarefas têm de ser alocadas às estações de trabalho e uma regra secundária para o desempate.

5) Delegação de tarefas, uma de cada vez, à primeira estação, até que a soma dos tempos seja igual ao tempo de ciclo ou até que nenhuma outra tarefa seja viável devido a restrições de tempo ou de sequência. Repetir o processo para as demais estações de trabalho até que todas as tarefas sejam alocadas.

6) Avaliar a eficiência da linha. A eficiência ou utilização é definida por Gaither e Frazier (2012) como o percentual de tempo que uma linha de produção opera. Pode ser obtida pelas Equações 3 ou 4.

$$
\begin{gathered}
U(\%)=\frac{\text { Número mínimo de estações de trabalho }}{\text { Número real de estações de trabalho }} \\
E(\%)=\frac{\text { Tempo total exigido para processar cada item }}{M T \times C}
\end{gathered}
$$

Conforme pode-se verificar também na Figura 1, a maioria das etapas descritas também fazem parte da heurística da utilização incremental. A metodologia para balancear a linha de montagem multiprodutos é a mesma da linha de um só produto, considerando-se como tempo de ciclo o tempo ponderado em função da quantidade a produzir de cada produto (MARTINS; LAUGENI, 2015).

De acordo com Gaither e Frazier (2012) a maioria das linhas de produção é rebalanceada diversas vezes por ano. Balancear novamente uma linha de produção significa incorrer na interrupção da produção o que pode gerar ociosidade de recursos. Entretanto, continuar operando uma linha de produção desbalanceada, com a capacidade incorreta entre as estações, pode gerar custos de produção elevados, além de estoques excessivos. A próxima seção apresenta o balanceamento de linha com programação linear.

Revista Produção Online. Florianópolis, SC, v.17, n. 3, p. 781-803, 2017. 


\subsection{Balanceamento de linha com programação linear}

Existem vários modelos matemáticos para o problema de arranjo físico (ANJOS; VIEIRA, 2017). Para comparar o desempenho da heurística da utilização incremental em relação à solução otimizada, o problema de balanceamento também pode ser resolvido, por exemplo, com a utilização da programação linear.

Seja $T_{i}$, o conjunto de tarefas designadas à estação $i, i=1, \ldots, m$, e $p_{j}$ o tempo de processamento da tarefa $j, j=1, \ldots, n$, então, a carga da estação $i$ é dada por $p\left(T_{i}\right)=\sum_{j \in T_{i}} p_{j}$. Quando um tempo de ciclo $c$ é dado, então uma linha balanceada terá $p\left(T_{i}\right)<c$, a estação $i$ tem um tempo ocioso de $c-p\left(T_{i}\right)$ unidades de tempo. O objetivo geral

é maximizar a eficiência da linha $E$, definida como $E=\frac{p_{\text {total }}}{m c}$, em que $p_{\text {total }}=\sum_{j=1}^{n} d_{j}$ (ARENALES et al, 2015).

\section{METODOLOGIA}

Este estudo caracteriza-se como de caráter exploratório e utilizou como trabalho de campo um estudo de caso. Segundo Voss (2002), o estudo de caso é uma investigação empírica que estuda um fenômeno contemporâneo em profundidade e em seu contexto natural, especialmente quando os limites entre o fenômeno e o contexto não são claramente evidentes.

Para verificar a eficácia da heurística da utilização incremental estudou-se uma empresa de serralheria que fabrica móveis escolares. É uma empresa nacional de grande porte que produz assentos esportivos e móveis escolares. Atua a mais de 30 anos no mercado e sua fábrica está localizada na cidade de Araruama no estado do Rio de Janeiro. No período do estudo, a empresa estava em fase de modernização dos seus equipamentos, substituindo processos manuais por processos automatizados. A primeira fase de modernização estava acontecendo no setor de serralheria para aumentar a capacidade produtiva do setor. O foco do estudo se deu no processo subsequente, ou seja, o de acabamento de superfície. 
Para a coleta dos dados foram realizadas visitas técnicas à empresa para entender seu processo produtivo desde a produção na serralheria até a montagem final dos móveis.

Gil (2009) descreve como utilizar as técnicas de coleta de dados no estudo de caso. Neste estudo foram utilizadas as seguintes:

- Observação espontânea;

- Entrevistas não estruturadas com os colaboradores, líderes e com o diretor industrial;

- Consulta dos relatórios de produção e das instruções de trabalho do setor de tratamento de superfície.

Por fim, propôs-se o balanceamento da linha de tratamento de superfície com a utilização da heurística da utilização incremental, comparando com a solução otimizada por programação linear, e verificou-se a viabilidade técnica das modificações no setor de serralheria.

\section{DESENVOLVIMENTO}

Conforme apontado, a empresa, objeto de estudo, no momento da coleta dos dados, estava modernizando o seu setor de serralheria. A modernização constitui basicamente a substituição de algumas operações realizadas pelos soldadores por células de solda. Com a realocação do trabalho, individual para estações, a empresa objetivava aumentar sua produção de 2500 para 3000 peças por dia.

O processo de fabricação da empresa era realizado conforme modelo de produção em massa, fornecendo produtos similares e sendo realizado em uma linha de produção. A Figura 2 apresenta o fluxograma com a sequência dos processos que compunham a produção. 
Figura 2 - Mapeamento do processo de produção

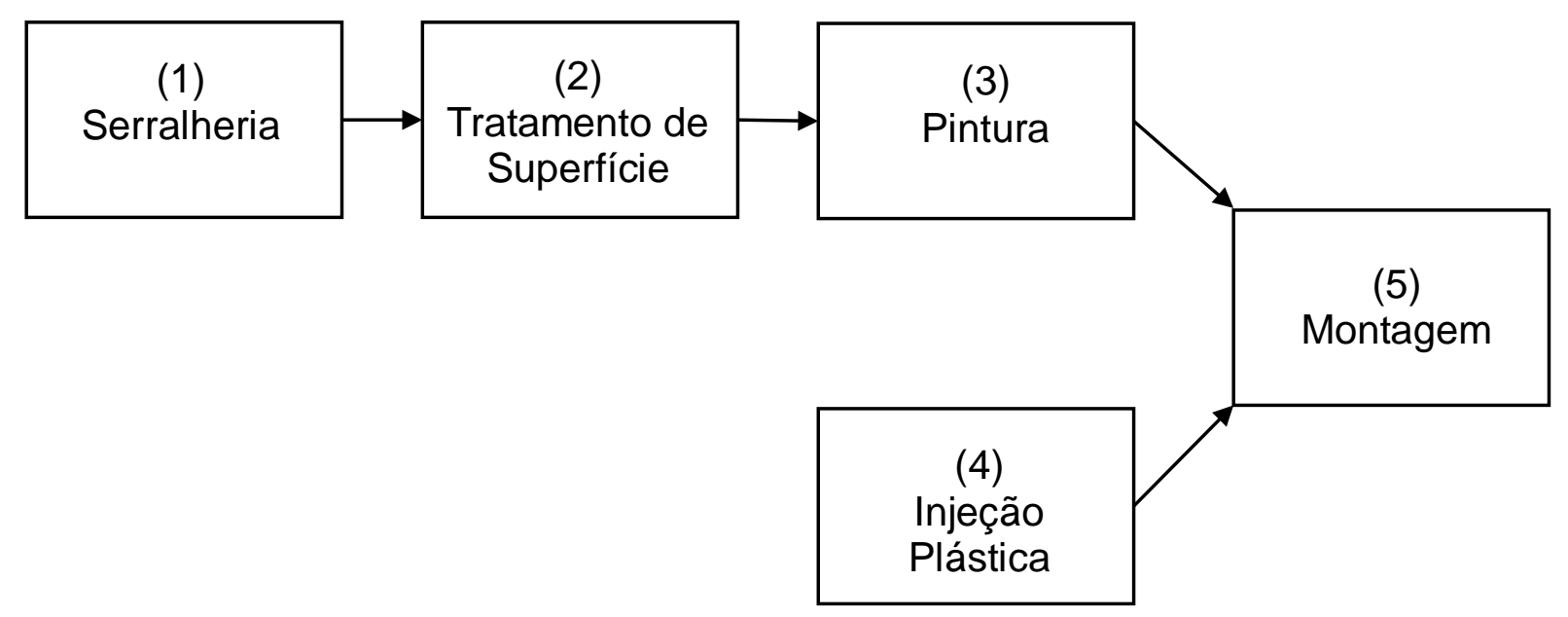

Fonte: elaborado pelos autores

Como pode ser visualizado na Figura 2, o aumento da produção da serralheria impactará o setor de tratamento de superfície. O setor de tratamento de superfície operava durante 24 horas por dia, 7 dias por semana em uma escala de $12 \mathrm{~h} \times 24 \mathrm{~h}$, ou seja, os colaboradores trabalham 12 horas e folgam $24 \mathrm{~h}$. Os dois turnos de produção têm um intervalo de $1 \mathrm{~h}$ para refeição dos colaboradores.

O processo de tratamento de superfície ocorre por imersão. A imersão das peças nos tanques é realizada através de cestos. A capacidade de peças por cesto varia de acordo com o produto, mas na média um cesto comporta 55 peças. Considerando-se que a jornada útil do setor de tratamento de superfície é de 22 horas por dia ou 1320 minutos por dia, sua capacidade de produção máxima seria de 47 cestos de peças por dia, ou 2585 peças.

A empresa precisava, portanto, aumentar a capacidade produtiva do setor de tratamento de superfície. O setor de tratamento de superfície é subsequente ao setor de serralheria e precisa absorver o novo volume de peças que seria produzido pelo setor de serralheria. Este setor é responsável pela preparação da peça antes do processo de pintura para garantir a aderência da tinta.

O processo produtivo da linha de produção analisada era constituído por nove tarefas: 
1. A primeira tarefa ou operação era o desengraxe que consistia na remoção de óleo e resíduos da peça, provenientes da operação de manufatura ou oleamento de usina, obtendo uma peça limpa, isenta de impurezas. Esta operação tinha duração de 10 minutos;

2. A segunda operação é o enxague para remoção dos resíduos da peça evitando a contaminação da operação consequente. Esta operação tinha duração de 1 minuto;

3. A terceira operação é a decapagem que consiste na remoção de camadas de ferrugem formada pela ação do tempo durante o transporte ou armazenamento. Esta operação tinha duração de 2 minutos;

4. A quarta operação é o enxague para remoção dos resíduos da peça provenientes do estágio de decapagem ácida, evitando a contaminação da operação consequente. Esta operação tinha duração de 1 minuto;

5. A quinta operação é o refinamento, cuja finalidade é condicionar a peça a ser fosfatizada para obtenção de uma camada de fosfato uniforme, densa e micro cristalina, evitando falhas ou imperfeições da camada de fosfato depositado;

6. A sexta operação é fosfatização que consiste na deposição sobre a peça de uma camada de fosfato;

7. A sétima operação é o enxague para remoção dos sais residuais da peça para evitar a contaminação da operação posterior. Esta operação tinha duração de 1 minuto;

8. A oitava operação é a passivação que consiste em selar as porosidades existentes na camada de fosfato;

9. A nona operação é o enxague para remoção dos sais solúveis residuais e do excesso de acidez para evitar a formação de blisters e focos de corrosão. Esta operação também duração de 1 minuto.

Revista Produção Online. Florianópolis, SC, v.17, n. 3, p. 781-803, 2017. 
A Figura 3 e a Tabela 1 resumem as operações, suas relações de precedência e o tempo de duração. A próxima seção apresenta a aplicação da heurística da utilização incremental.

Figura 3 - Diagrama de precedência do processo do setor de tratamento de superfície

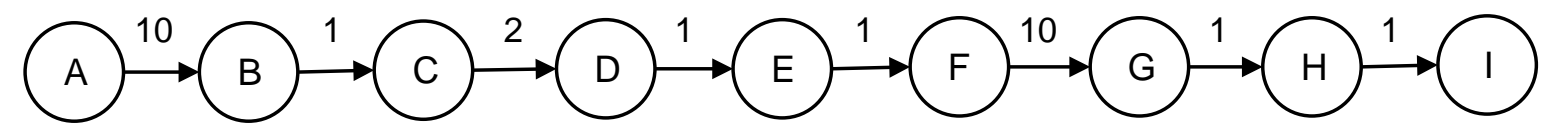

Fonte: elaborado pelos autores

Tabela 1 - Operações do processo de tratamento de superfície

\begin{tabular}{cc}
\hline Operações & Descrição \\
\hline A & Desengraxe \\
B & Enxague \\
C & Decapagem \\
D & Enxague \\
E & Refinamento \\
F & Fosfatização \\
G & Enxague \\
H & Passivação \\
I & Enxague \\
\hline
\end{tabular}

Fonte: elaborado pelos autores

\subsection{Aplicação da heurística}

Em um levantamento preliminar, verificou-se que a linha de produção analisada atendia as hipóteses necessárias para que o problema de balanceamento de linha fosse considerado aplicável. Apesar da linha de tratamento de superfície atender diversos modelos de móveis, os tempos de operações eram iguais para todos os produtos.

O balanceamento da linha de tratamento de superfície foi realizando utilizandose as seis etapas descritas por Davis, Aquilano e Chase (2001) e o método heurístico de utilização incremental, apresentado na Figura 1. Assim como destacado, o método para melhoria do balanceamento de linha baseado na regra da heurística de utilização incremental considera que seja inicialmente determinado o tempo de ciclo.

Revista Produção Online. Florianópolis, SC, v.17, n. 3, p. 781-803, 2017. 
O cálculo do tempo de ciclo foi baseado numa produção de 22 horas diárias ou 1320 minutos (dois turnos de 12 horas, subtraído de 1 hora de folga para refeições em cada turno), dividido por uma produção de 3000 unidades por dia ou 55 cestos (cada cesto comporta em média 55 peças). Desta forma, aplicando-se a Equação 1, tem-se o seguinte resultado do tempo de ciclo calculado exibido na Equação 5:

$$
c=\frac{1320}{55}=24
$$

Após o cálculo do tempo de ciclo, pôde-se calcular o número mínimo teórico de estações de trabalho. Sabendo-se que o tempo total para produzir uma peça na linha é igual a soma dos tempos de todas as tarefas envolvidas no processo produtivo, o número mínimo teórico de estações pode ser exibido na Equação 6.

$$
M T=\frac{28}{24}=1,1667
$$

Como o número mínimo teórico de estações de trabalho deve ser inteiro, obtémse 2 estações de trabalho. Em seguida, determinou-se a ordem de alocação das tarefas. Isto foi feito seguindo-se a sequência de tarefas e a regra heurística de utilização incremental, usada para agrupar as tarefas em centros de trabalho. Nesse método, as tarefas são combinadas em sequência até que a utilização do centro de trabalho seja $100 \%$ ou até que se observe que a utilização do centro de trabalho diminua, e então um novo centro de trabalho é iniciado.

$O$ centro de trabalho 1 considera primeiro a tarefa $A$ isoladamente com taxa de utilização de $41,76 \%$. Considerando-se em seguida as tarefas A e B juntas, a utilização do centro de trabalho se eleva para 45,83\%. O aumento da utilização do centro de trabalho acontece até o agrupamento das tarefas $A, B, C, D$ e E, pois, quando a tarefa F é adicionada a A, B, C, D e E, a utilização cai de $62,5 \%$ para $52,08 \%$. O centro de trabalho 1 incorpora então as tarefas A, B, C, D e E. A Tabela 2 ilustra o resultado das alocações das tarefas.

Revista Produção Online. Florianópolis, SC, v.17, n. 3, p. 781-803, 2017. 
Tabela 2 - Alocação das tarefas

\begin{tabular}{cccccc}
\hline $\begin{array}{c}\text { Centro } \\
\text { de } \\
\text { Trabalho }\end{array}$ & Tarefa & $\begin{array}{c}\text { Tempo de } \\
\text { duração da } \\
\text { operação } \\
\text { (min) }\end{array}$ & $\begin{array}{c}\text { Tempo das estações de } \\
\text { trabalho agregada dividido } \\
\text { pelo tempo de ciclo }\end{array}$ & $\begin{array}{c}\text { Número } \\
\text { Real de } \\
\text { Estações }\end{array}$ & Utilização \\
\hline $\mathbf{1}$ & A & 10,00 & 0,416667 & 1 & $41,67 \%$ \\
$\mathbf{1}$ & A,B & 11,00 & 0,458333 & 1 & $45,83 \%$ \\
$\mathbf{1}$ & A,B,C & 13,00 & 0,541667 & 1 & $54,17 \%$ \\
$\mathbf{1}$ & A,B,C,D & 14,00 & 0,583333 & 1 & $58,33 \%$ \\
$\mathbf{1}$ & A,B,C,D,E & 15,00 & 0,625 & 1 & $62,50 \%$ \\
$\mathbf{1}$ & A,B,C,D,E,F & 25,00 & 1,041667 & 2 & $52,08 \%$ \\
$\mathbf{2}$ & F & 10,00 & 0,416667 & 1 & $41,67 \%$ \\
$\mathbf{2}$ & F,G & 11,00 & 0,458333 & 1 & $45,83 \%$ \\
$\mathbf{2}$ & F,G,H & 12,00 & 0,5 & 1 & $50,00 \%$ \\
$\mathbf{2}$ & F,G,H,I & 13,00 & 0,541667 & 1 & $54,17 \%$ \\
\hline
\end{tabular}

Fonte: elaborado pelos autores

A designação de tarefas a estações de trabalho na linha de tratamento de superfície é resumida na Tabela 3.

Tabela 3 - Designação de tarefas a estações de trabalho

\begin{tabular}{lcc}
\hline Tarefas em centro de trabalho & $\mathrm{A}, \mathrm{B}, \mathrm{C}, \mathrm{D}, \mathrm{E}$ & $\mathrm{F}, \mathrm{G}, \mathrm{H}, \mathrm{I}$ \\
\hline Centros de trabalho & 1 & 2 \\
Número real de estações de trabalho & 1 & 1 \\
Tempo consumido & 15 & 13 \\
Tempo Disponível & 24 & 24 \\
\hline
\end{tabular}

Fonte: elaborado pelos autores

Por fim, calculou-se a eficiência do balanceamento pela utilização da Equação 4 e exibido na Equação 7.

$$
E(\%)=\frac{28 \times 100}{2 \times 24}=58,33 \%
$$

Com a linha de produção balanceada, calculou-se novamente a capacidade produtiva da linha de produção que passou de 2585 para 4840 peças. A partir dos resultados do estudo, a empresa decidiu por dar continuidade ao processo de modernização do setor de serralheria, uma vez que serão necessários poucos ajustes no setor de tratamento de superfície para o aumento da sua produtividade, aumentando apenas uma estação de trabalho. Deste modo, a empresa considerou incluir o resultado 
do estudo no seu planejamento. A próxima seção apresenta o resultado obtido com programação linear.

\subsection{Resolução com programação linear}

A solução para o balanceamento de linha obtida pela aplicação da regra da heurística da utilização incremental, descrita na seção anterior, foi comparada com a solução otimizada com programação linear de modo a avaliar a eficácia da heurística da utilização incremental.

O processo de modelagem apresentado na seção 2.3 foi adaptado, conforme Mayerle (2015), para aplicação no Microsoft Excel@.

Assim como no balanceamento realizado por meio de heurísticas, foi determinado inicialmente o número de estações conforme a Equação 8:

$$
m=\frac{\sum p}{c}
$$

Onde:

$m$ - quantidade de estações

$p$ - tempo de processamento

$c$ - tempo de ciclo

Após a definição da quantidade de estações, aplicaram-se penalidades $a_{i}$ de modo que a otimização buscasse atribuir o número máximo de tarefas a uma única estação, limitado ao tempo de ciclo. Houve também a necessidade de que as penalidades fossem crescentes a partir da primeira estação, de modo que, $a_{i}<a_{i+1}<, \ldots,<a_{m}$. As células variáveis, $X_{i}, \ldots, X_{m}$, representaram as estações.

O tempo total, dado pela multiplicação das estações com seus tempos de processamento, foi obtido pela Equação 9.

$$
\sum_{i=1}^{m} \sum_{j=1}^{n} X_{i} p_{j}
$$

Revista Produção Online. Florianópolis, SC, v.17, n. 3, p. 781-803, 2017. 
O problema é então formulado como:

$$
\text { Minimizar } \sum_{i=1}^{m} X_{i} a_{i}
$$

Sujeito a

$$
\begin{aligned}
& \sum_{i=1}^{m} \sum_{j=1}^{n} X_{i} p_{j} \leq c \\
& X_{i-1} \leq X_{i}, \quad \forall i \\
& \sum_{i=1}^{m} X_{i}=1 \\
& X_{i} \in B^{n}
\end{aligned}
$$

A função objetivo (10) minimiza a quantidade de estações. As restrições (11) e (12) consideraram a limitação no tempo de ciclo e o número de estações. A restrição (13) garante que cada tarefa seja atribuída a apenas uma estação. A restrição (14) representa o tipo das variáveis. Os resultados são apresentados na Tabela 4.

Tabela 4 - Resultado do balanceamento com otimização linear

\begin{tabular}{cccc}
\hline \multirow{2}{*}{ Tarefa } & \multirow{2}{*}{ Tempo } & $\mathbf{1}$ & $\mathbf{2}$ \\
\hline A & 10 & 1 & 0 \\
B & 1 & 1 & 0 \\
C & 2 & 1 & 0 \\
D & 1 & 1 & 0 \\
E & 1 & 1 & 0 \\
F & 10 & 0 & 1 \\
G & 1 & 0 & 1 \\
H & 1 & 0 & 1 \\
I & 1 & 0 & 1 \\
& Penalidade & $\mathbf{1}$ & $\mathbf{1 0}$ \\
\hline
\end{tabular}

Fonte: elaborado pelos autores

Conforme pode-se verificar pela análise da Tabela 4, a solução otimizada resultou na mesma alocação determinada pela heurística da utilização incremental. As 
tarefas A, B, C, D e E, foram atribuídas à estação 1 e as tarefas restantes à estação 2. A próxima seção apresenta a discussão dos resultados.

\section{DISCUSSÃO}

Os resultados do estudo apontaram a adequação da heurística da utilização incremental para aumentar a eficiência de uma linha de produção por meio do balanceamento das atividades. Conforme apontado por Gaither e Frazier (2012), a heurística da utilização incremental foi eficaz para balancear uma linha de produção por produto, aumentando sua eficiência.

O fato do resultado proposto pela heurística da utilização incremental ter obtido o mesmo resultado da proposição realizada com a programação linear reforça a eficácia deste método heurístico e simplificado para balancear linhas de produção. O aumento da produção obtido após o balanceamento também suporta os argumentos de Silva e Rentes (2012) e Tompkins et al. (2003), acerca da capacidade do método.

Outra peculiaridade importante destacada neste estudo pode ser elencada como a vantagem da regra da heurística incremental em resolver problemas de balanceamento de linha independentemente da duração das tarefas em relação ao tempo de ciclo total conforme apontou Gaither e Frazier (2012). Esse fato pode ser comprovado pela quantidade de tarefas que tinham tempo de processamento semelhantes (1 minuto).

Quanto à programação linear realizada, o modelo formulado por Arenales et al. (2015) e operacionalizado por Mayerle (2015) se mostraram úteis para identificar a solução ótima para o problema de balanceamento. Neste estudo, o modelo foi eficaz ao avaliar a eficácia da heurística da utilização incremental por permitir a comparação com a solução ótima.

Por fim, o estudo contribuiu com a temática da utilização de regras heurísticas para balanceamento de linha, ao avaliar a heurística da utilização incremental e comparar seus resultados com a solução ótima. Tal como fez Breginski (2013), ao 
comparar diversas heurísticas, o estudo pode se tornar referências para futuras comparações.

\section{CONSIDERAÇÕES FINAIS}

O presente artigo tratou do problema de balanceamento de linha de montagem simples que tem como objetivo a minimização do número de estações de trabalho (SALBP-1). Os resultados obtidos confirmaram a hipótese de que a heurística da utilização incremental foi eficaz para balancear a linha de tratamento de superfície, que tinha disposição em layout por produto, aumentando a sua produtividade permitindo que o setor analisado absorva a nova capacidade de produção da seção precedente, que é a de serralheria.

O trabalho contribuiu de maneira teórica e prática à medida que realizou uma aplicação prática de resolução de problema de balanceamento de linha de montagem, auxiliando na decisão da empresa de aumentar a capacidade produtiva do setor de serralheria. A análise dos resultados obtidos torna evidente a importância do balanceamento como forma de melhorar o processo produtivo da linha de tratamento de superfície da empresa.

Com relação à heurística utilizada, conclui-se que consiste num método simples e prática de ser utilizado e foi capaz de desenvolver uma solução para o problema. Com relação à aplicação prática da regra, no estudo de caso, verificou-se que, com o balanceamento do setor de tratamento de superfície foi possível absorver a nova capacidade produtiva do setor de serralheria ressaltando resultados benéficos com o uso de uma metodologia útil.

Apesar dos resultados apresentados não poderem ser generalizados, a partir de um estudo de caso, a relevância dessa pesquisa é dada pela aplicação prática do problema de balanceamento de linha. Esta foi uma lacuna apontada por vários autores, como: MELLER e GAU (1996); EREL e SARIN (1998); SCHOLL e BECKER (2006); BOYSEN et al (2007); SILVA et al (2014).

Revista Produção Online. Florianópolis, SC, v.17, n. 3, p. 781-803, 2017. 
O estudo propõe uma solução para o problema de balanceamento de linha de forma antecipada, tendo em vista a necessidade de ampliação da capacidade de produção da fábrica. Entretanto, vale ressaltar que o problema se dá inicialmente em um ambiente de capacidade superior à demanda, dificultando uma avaliação apropriada da vantagem do uso da heurística sobre outras abordagens.

Pesquisas futuras, no âmbito estudado, podem realizar o balanceamento da linha de tratamento de superfície considerando postos de trabalho paralelos para as operações de desengraxe e fosforização. Em outro contexto, pode-se avaliar a eficácia da heurística da utilização incremental em outros tipos de arranjos físicos.

\section{REFERÊNCIAS}

ANJOS, Miquel F.; VIEIRA, Manuel VC. Mathematical optimization approaches for facility layout problems: The state-of-the-art and future research directions. European Journal of Operational Research, 2017. https://doi.org/10.1016/j.ejor.2017.01.049

ARENALES, M.; ARMENTANO, V.; MORABITO, R.; YANASSE, H. Pesquisa operacional: para cursos de engenharia. 2. ed. Rio de Janeiro: Elsevier, 2015.

BOYSEN, N., FLIEDNER, M.; SCHOLL, A. A classification of assembly line balancing problems. European Journal of Operational Research, v.183, p. 674-693, 2007. https://doi.org/10.1016/j.ejor.2006.10.010

BREGINSKI, R.B. Balanceamento e sequenciamento de linhas de montagem de modelo misto: um estudo de caso da indústria automotiva no Brasil. 114 f. Dissertação (Mestrado em Engenharia de Produção) - Universidade Federal do Paraná - UFPR, Curitiba, 2013.

CRISTO, R.L. Balanceamento de linhas de montagem com uso de algoritmo genético para o caso de linhas simples e extensões. 83 f. Dissertação (Mestrado em Engenharia de Produção) - Universidade Federal de Santa Catarina- UFSC, Florianópolis, 2010.

DAVIS, M.; AQUILANO, N.; CHASE, R. Fundamentos da administração da produção. 3. ed. Porto Alegre: Bookman, 2001.

Drira, A.; Hajri-Gabouj, S.; Pierreval, H. Facility layout problems: A survey. Annual Reviews in Control, v. 31, p. 255-267, 2007. https://doi.org/10.1016/j.arcontrol.2007.04.001

EREL, E.; SARIN, S.C. A survey of the assembly line balancing procedures, Production Planning \& Control, v. 9, n. 5, p. 414-434, 1998. http://dx.doi.org/10.1080/095372898233902 
FARNES, V. C. F., PEREIRA, N. A. Balanceamento de linha de montagem com o uso de heurística e simulação: estudo de caso na linha branca. Gestão da Produção, Operações e Sistemas, v. 2, p. 125- 136, 2006.

FERNANDES, F.C. DALALIO, A.G. Balanceamento e rebalanceamento de linhas de montagem operadas por grupos de trabalho autogerenciados. Gest. Prod., São Carlos, v. 7, n. 3, p. 378398, 2000. http://dx.doi.org/10.1590/S0104-530X2000000300013.

GAITHER, N.; FRAZIER, G. Administração da produção e operações. 8. ed. São Paulo: Cengage, 2012.

GIL, A. C. Como elaborar projetos de pesquisa. 4. ed. São Paulo: Atlas, 2009.

GOSH, S.; GAGNON, R. J. A comprehensive literature review and analysis of the design, balancing and scheduling of assembly systems. International Journal of Production Research, v. 27, p. 637- 670, 1989. http://dx.doi.org/10.1080/00207548908942574

HELGESON, W. B.; SALVESON, M. E.; SMITH, W. W. How to balance an assembly line. Management Report, v. 7, 1954.

Kumar, D. M. Assembly line balancing: a review of developments and trends in approach to industrial application. Global Journal of Research In Engineering, v. 13, n. 2, 2013. http://dx.doi.org/10.17406/gire

MAKE, Muhammad Razif Abdullah; RASHID, Mohd Fadzil Faisae Ab; RAZALI, Muhamad Magffierah. A review of two-sided assembly line balancing problem. The International Journal of Advanced Manufacturing Technology, v. 89, n. 5-8, p. 1743-1763, 2017. http://dx.doi.org/10.1007/s00170-016-9158-3

MARTINS, P.G. LAUGENI, F.P. Administração da produção. 3. ed. São Paulo: Saraiva, 2015. MAYERLE, S. Pesquisa operacional. Apostila de curso. 2015.

MELLER, R. D.; GAU, K. Y. The facility layout recent and emerging trends and perspectives. Journal of Manufacturing Systems, v. 15, n. 5, p. 351-366, 1996.

https://doi.org/10.1016/0278-6125(96)84198-7

OLIVEIRA, ISAAC MESSIAS DINIZ ; DA PAZ, CARLA CANHA ; DA SILVA, ADRIANO MANIÇOBA ; FERREIRA, WILLIAM DE PAULA. Balanceamento de linha e arranjo físico: estudo de caso em uma linha de produção de cabines para máquinas de construção. Exacta (Online), v. 15, p. 101-110, 2017. http://doi.org/10.5585/ExactaEP.v15n1.6697

REGINATO, G. Métodos de balanceamento de linhas de montagem uni-modelo e mista. 71 f. Dissertação (Mestrado em Engenharia de Produção) - Universidade Federal do Rio Grande do Sul - UFRGS, Porto Alegre, 2014. 
SALVESON, Melvin E. The assembly line balancing problem. Journal of Industrial Engineering, v. 6, n. 3, p. 18-25, 1955.

SANTOS, Luciano Costa; DE MIRANDA ARNAUD, Leonardo; DUTRA, Jéssica Bezerril. Uma dinâmica para o ensino da matriz produto-processo: roteiro e aplicação. Revista Produção Online, v. 14, n. 3, p. 1129-1150, 2014. http://dx.doi.org/10.14488/1676-1901.v14i3.1700

SCHOLL, A.; BECKER, C. State-of-the-art exact and heuristic solution procedures for simple assembly line balancing. European Journal of Operational Research, v.168, n.3, p.666-693, 2006. http://dx.doi.org/10.1016/j.ejor.2004.07.022

SILVA JUNIOR, O. C.; MIRANDA JUNIOR, G.; CONCEICAO, S. V. Reconfiguração dinâmica de linhas de montagem. Pesqui. Oper., Rio de Janeiro , v. 30, n. 1, p. 237-258, 2010. http://dx.doi.org/10.1590/S0101-74382010000100012

SILVA, A. L.; RENTES, A. F. Um modelo de projeto de layout para ambientes job shop com alta variedade de peças baseado nos conceitos da produção enxuta. Gest. Prod., São Carlos, v. 19, n. 3, p. 531-541, 2012. http://dx.doi.org/10.1590/S0104-530X2012000300007

SILVA, G. G. M. P.; TUBINO, D. F., SEIBEL, S. Linhas de Montagem: revisão da literatura e oportunidades para pesquisas futuras. Prod., São Paulo, v. 25, n. 1, p. 170-182. 2014. http://dx.doi.org/10.1590/S0103-65132014005000001

SIVASANKARAN, P., SHAHABUDEEN, P. Literature review of assembly line balancing problems. The International Journal of Advanced Manufacturing Technology, v. 73, n. 9/12, 1665-1694, 2014. http://dx.doi.org/10.1007/s00170-014-5944-y

TELO, LEONARDO ROSSI AZEVEDO ; SILVA, RAPHAEL MARTINS DA ; SILVA, ADRIANO MANICCOBA DA ; ZAMPINI, EUGENIO DE FELICE ; PAULA, WILLIAM FERREIRA DE . Proposição de sequenciamento da produção com uso do tempo de preparação. South American Development Society Journal, v. 3, p. 19, 2017. http://dx.doi.org/10.24325/issn.2446-5763.v3i8p29-49

TOMPKINS, J.A. et al. Facilities planning. 3. Ed. New York: Jonh Wiley \& Sons, 2003.

SNATKIN, Aleksei et al. Production monitoring system development and modification. Proceedings of the Estonian Academy of Sciences, v. 64, n. 4, p. 567, 2015. http://dx.doi.org/10.3176/proc.2015.4S.04

VOSS, Chris; TSIKRIKTSIS, Nikos; FROHLICH, Mark. Case research in operations management. International journal of operations \& production management, v. 22, n. 2, p. 195-219, 2002. https://doi.org/10.1108/01443570210414329

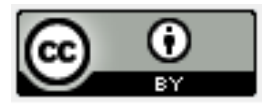

Artigo recebido em 14/06/2016 e aceito para publicação em 21/08/2017

DOI: http://dx.doi.org/10.14488/1676-1901.v17i3.2404

Revista Produção Online. Florianópolis, SC, v.17, n. 3, p. 781-803, 2017. 